\title{
Therapeutic Effect of Idebenone on Rats with Vascular Dementia via the MicroRNA-2I6a/RSK2/ NF- $\kappa B$ Axis
}

This article was published in the following Dove Press journal: Neuropsychiatric Disease and Treatment

\section{Xudong Qian' \\ Qianqian $\mathrm{Xu}^{\prime}$ \\ Guoyun $\mathrm{Li}^{2}$ \\ Yi Bu' \\ Fan Sun' \\ Jian Zhang'}

'Department of Neurology, Affiliated Hospital of Chengde Medical University, Chengde, 067000, Hebei, People's Republic of China; ${ }^{2}$ Department of Respiratory, Affiliated Hospital of Chengde Medical University, Chengde, 067000, Hebei, People's Republic of China
Correspondence: Jian Zhang Department of Neurology, Affiliated Hospital of Chengde Medical University, No. 36, Nanyingzi Street, Chengde, 067000, Hebei, People's Republic of China $\mathrm{Tel} / \mathrm{Fax}+86$ 03।4-2279965

Email Zhangjian8I4I@I63.com
Background: Vascular dementia (VD) is a brain disease featured by cognitive impairment and cerebrovascular pathologies. Idebenone can treat neurodegenerative diseases. This study evaluated the mechanism of Idebenone in VD.

Methods: The VD rat model was established by permanent occlusion of bilateral common carotid arteries, followed by intragastrical administration of Idebenone. The learning and spatial memory abilities, and the levels of MDA, SOD, IL- 6 and TNF- $\alpha$ were measured. Histological staining was adopted to observe the damage of neurons in the hippocampal cortex and to quantitatively analyze the neuronal damage in CA1 area of hippocampus. Microarray analysis was performed to find out the effect of Idebenone treatment on microRNA (miR) expression in hippocampus of rats. The potential target genes of miR and the pathways regulated by target genes were searched by bioinformatics analysis, and verified by experiments. The mechanism of action behind Idebenone in VD rats was proved by rescue experiment.

Results: Idebenone treatment improved the learning and spatial memory abilities of VD rats, inhibited neuroinflammation and oxidative stress, and prevented neuronal apoptosis. Idebenone treatment elevated miR-216a expression in hippocampus of rats, but the therapeutic effect of Idebenone was averted by lentivirus inhibition of miR-216a. miR-216a targeted RSK2. Overexpression of RSK2 annulled the therapeutic effect of Idebenone on $\mathrm{VD}$ rats by activating the $\mathrm{I} \kappa \mathrm{B} \alpha / \mathrm{NF}-\kappa \mathrm{B}$ axis.

Conclusion: Idebenone inhibits the activation of $\mathrm{RSK} 2 / \mathrm{I} \kappa \mathrm{B} \alpha / \mathrm{NF}-\kappa \mathrm{B}$ axis by increasing miR-216a, thus alleviating oxidative stress and neuroinflammation in VD rats.

Keywords: vascular dementia, idebenone, microRNA-216a, RSK2, I $\mathrm{B} \mathrm{B} / \mathrm{NF} \kappa \mathrm{B}$, oxidative stress

\section{Introduction}

Vascular dementia (VD) is a heterogeneous brain disease, which is featured by cognitive impairment and cerebrovascular pathologies, with the incidence rate of at least $20 \%$ of dementia second to Alzheimer's disease. ${ }^{1}$ In clinical, VD is manifested as cognitive, psychological and behavioral disorders, such as repeated questioning, irritability, depression, apathy, aggression, sleep disorders and other adverse behaviors. ${ }^{2}$ The common etiologies of VD include large artery atherosclerosis, small vessel disease, myogenic stroke and cardiovascular disorders. ${ }^{3}$ A variety of cellular signaling and regulatory mechanisms are implicated in $\mathrm{AD}$, including apoptosis, oxidative stress and inflammation. ${ }^{4}$ Oxidative stress has been confirmed to be related to the 
pathogenesis and risk factors of VD, and antioxidant therapy might play a role in the management of VD. ${ }^{5}$ Moreover, the cognitive impairment is attributed to both vascular disease and neuroinflammation. ${ }^{6}$ At present, cholinesterase inhibitors are recognized as available therapeutic agents for VD, but these drugs may produce serious adverse reactions, such as gastrointestinal reactions, joint pain, dizziness and headache, while their ability to improve the patient remains to be determined. ${ }^{7}$ With the increasing proportion of the elderly, elucidating the molecular mechanism of VD and developing potent agents are urgent for improving the quality of life for patients and reducing the social burden.

Idebenone is an analogue of coenzyme Q10 (CoQ10), which is a lipophilic electron carrier and endogenous antioxidant existing in all cell mitochondrial membranes. ${ }^{8}$ As a kind of benzoquinone that can produce reversible redox reaction, Idebenone affects the electron balance of mitochondria. ${ }^{9}$ In vitro research has revealed that Idebenone protects mitochondrial function against amyloid beta toxicity in primary cortical neurons. $^{10}$ In view of its mechanism in mitochondria, Idebenone is extensively used to treat neurodegenerative diseases and diseases associated with mitochondrial etiology. ${ }^{11}$ A previous study has demonstrated that the cognitive status of patients with cerebrovascular disease and degenerative disorders can be notably improved after receiving Idebenone treatment. ${ }^{12}$ However, whether Idebenone is involved in alleviating VD by regulating oxidative stress and neuroinflammation remains unclear.

MicroRNAs (miRs) are attractive molecules that can be used as one of the biomarkers of neurodegenerative diseases. ${ }^{13}$ For example, miR-216a upregulation is neuroprotective against ischemic injury by inhibiting apoptosis and inflammation. $^{14}$ Importantly, miR-216a inhibits neuronal apoptosis in a cell model of Parkinson's disease. ${ }^{15}$ But the role of miR-216a in VD remains to be established. This study explored the effect of Idebenone on oxidative stress and neuroinflammation in VD, along with its miR mechanism, which shall shed lights on the development of pharmacotherapy for VD.

\section{Materials and Methods}

\section{Ethics Statement}

All animal protocols were strictly on the basis of the National Institutes of Health Guide for the Care and Use of Laboratory Animals. The animal ethics committee of Affiliated Hospital of Chengde Medical University approved all protocols and experimental designs. All efforts were made to minimize the animal suffering.

\section{Animal Model}

Healthy adult Sprague-Dawley male rats (specificpathogen-free grade, 250-300g, permit number: SCXK (Xiang) 2019-0004) were obtained from Hunan SJA Laboratory Animal Co., Ltd. (Changsha, Hunan, China). The rats were kept in a 12 -h light/dark cycle at $25 \pm 1{ }^{\circ} \mathrm{C}$.

According to a previous report, ${ }^{16}$ a VD rat model was established by permanent occlusion of bilateral common carotid arteries (CCA). Within $12 \mathrm{~h}$ before the operation, all rats were fasted with free access to water. Each rat was anesthetized with a mixture of 5\% isoflurane and $95 \%$ oxygen, and then a mixture of $3 \%$ isoflurane and $97 \%$ oxygen was supplied through a breathing mask during the operation to maintain anesthesia. The rats were placed on the electrothermal pad to maintain the anal temperature at $37^{\circ} \mathrm{C}$. For VD rats, a $1.5-2 \mathrm{~cm}$ incision was made on the epidermis along the midline of the neck to separate the muscles until the common carotid artery was exposed. Two segments of CCA were ligated using No.0 silk thread, and the middle artery was blocked by electrocoagulation. The other side of the CCA was treated similarly. The sham-operated rats were treated the same as that in VD rats, but the CCA was not ligated or blocked. To avoid potential postoperative infection, the wound was washed with $20 \mathrm{mg} / \mathrm{mL}$ gentamicin sulfate solution (B540724, Sangon Biotech Co., Ltd., Shanghai, China) before wound closure. The wound was then sutured to allow the rats to recover from anesthesia and return to the cage.

There were 10 sham-operated rats and 50 VD modeling rats. Fifteen days after surgery, VD rats were arbitrarily assigned into VD group, Idebenone group (oral administration of Idebenone at $100 \mathrm{mg} / \mathrm{kg}$ daily for 3 weeks), lentiviral vector negative control ( $\mathrm{Lv}-\mathrm{NC})$ group (after Idebenone administration, empty Lv-NC was injected at the 16th day after surgery), Lv-inhibitor group (after Idebenone administration, Lv inhibitor of miR-216a was injected at the 16th day after surgery), and Lv-RSK2 group (after Idebenone administration, overexpression Lv of RSK2 was injected at the 16 th day after surgery), with 10 rats in each group.

Idebenone (catalogue number: HY-N0303, purity: 99.62\%, CAS No.58186-27-9) was purchased from Medchemexpress (Monmouth Junction, NJ, USA). The lentivirus for injection was purchased from Shanghai GenePharma Co., Ltd. (Shanghai, China) with a titer of 1 $\times 10^{9} \mathrm{TU} / \mathrm{mL}$. According to the previous report, ${ }^{17}$ lentivirus 
was injected into the CA1 region of the hippocampus. First, the rats were anesthetized by the method described previously and then placed into a stereotactic apparatus (Yuyan Instrument, Shanghai, China). Injection coordinates relative to the bregma were anteroposterior, $-4.52 \mathrm{~mm}$; mediolateral, $\pm 3.2 \mathrm{~mm}$; dorsoventral $-3.16 \mathrm{~mm}$ below the surface of dura using coordinates derived from the atlas of Paxinos and Watson (1997). Two $\mu \mathrm{L}$ lentiviral vector was injected into CA1 region of hippocampus using a $5 \mu \mathrm{L}$ syringe with a 33-gauge tip needle (Hamilton). The needle was maintained in this position for $2 \mathrm{~min}$ and then withdrawn slowly to prevent solution backflow. The accuracy of injection sites was identified by stereotaxic injection of Evans blue (Sigma-Aldrich, Merck KGaA, Darmstadt, Germany) into CA1 subregion of hippocampus.

The Morris water maze (MWM) test was performed from the 36 th to 42 nd days after surgery to test the learning and spatial memory abilities of the rats. On day 43, rats were euthanized with an overdose of pentobarbital sodium $(200 \mathrm{mg} / \mathrm{kg})$. The specific experimental process is shown in Figure 1.

\section{MWM Test}

The water maze device is a black circular water tank with a diameter of $120 \mathrm{~cm}$, a height of $50 \mathrm{~cm}$ and a water depth of $30 \mathrm{~cm}$. A black platform with a diameter of $10 \mathrm{~cm}$ was placed in the water maze through the recording system (SMART Panlab, Harvard Bioscience, Shanghai, China). The top of the platform was about $2 \mathrm{~cm}$ below the water surface. The tank was divided into 4 equal parts (A-D areas) and platform area ( $\mathrm{T}$ area). The platform was located in area A. In stage I (training phase, at day 36), each rat was gently placed in a water maze in area $\mathrm{C}$ to face the wall for $90 \mathrm{~s}$. If the platform was not found during the training, the rats were guided to the platform and maintained for $20 \mathrm{~s}$ for observation and learning. In stage II (during day 37-day 41), each rat was gently placed in the water maze in area $\mathrm{C}$ to face the water tank wall and look for the platform. If the rats successfully found the platform and stayed in the T area for $2 \mathrm{~s}$, the time was recorded. If the platform was not found within 90 $\mathrm{s}$, the time was recorded as $90 \mathrm{~s}$, and then the rats were guided to the platform and maintained for $20 \mathrm{~s}$ for observation and learning. In stage III (platform crossover phase, at day 42), the platform was removed, and each rat was gently placed in the water maze in area $\mathrm{C}$. The number of times the rats crossed the $\mathrm{T}$ area in $90 \mathrm{~s}$ was recorded. The learning ability and spatial memory ability of rats were evaluated based on the latency of platform searching and the times of platform crossing.

\section{Enzyme-Linked Immunosorbent Assay (ELISA)}

The hippocampal tissues were collected and ground with the precooled homogenate buffer solution ( $137 \mathrm{mM} \mathrm{NaCl}, 20 \mathrm{mM}$ Tris, 1\% NP40, 10\% glycerol, $1 \mathrm{mM}$ PMSF, $10 \mu \mathrm{g} / \mathrm{mL}$ aprotinin, $1 \mu \mathrm{g} / \mathrm{mL}$ leupeptin, $0.5 \mathrm{mM}$ sodium vanadate) with a tissue masher (Changzhou, Jiangsu, China) at 10,000-15,000 $\mathrm{r} / \mathrm{min}$. Then, the tissue homogenate was centrifuged in a lowtemperature and low-speed centrifuge (Beckman, Chaska, $\mathrm{MN}, \mathrm{USA}$ ) at $3000 \mathrm{r} / \mathrm{min}$ for $10-15 \mathrm{~min}$ to obtain the supernatant. ELISA kits were adopted to measure the concentration of interleukin (IL)-6 (ab234570, Abcam, Cambridge, MA, USA), tumor necrosis factor- $\alpha$ (TNF- $\alpha$, ab46070, Abcam), malondialdehyde (MDA, A003-1-2, Nanjing Jiancheng Bioengineering Institute, Nanjing, China) and superoxide dismutase (SOD, A001-3-2, Nanjing Jiancheng) in the supernatant of hippocampus tissue homogenate.

\section{Hematoxylin and Eosin (HE) Staining}

The brain tissues of rats were embedded in paraffin and sectioned. Paraffin-embedded sections $(4 \mu \mathrm{m})$ were dewaxed in xylene and hydrated with ethanol. After washing three times with phosphate-buffered saline (PBS), each tissue section was stained with $100 \mu \mathrm{L}$ pre-prepared

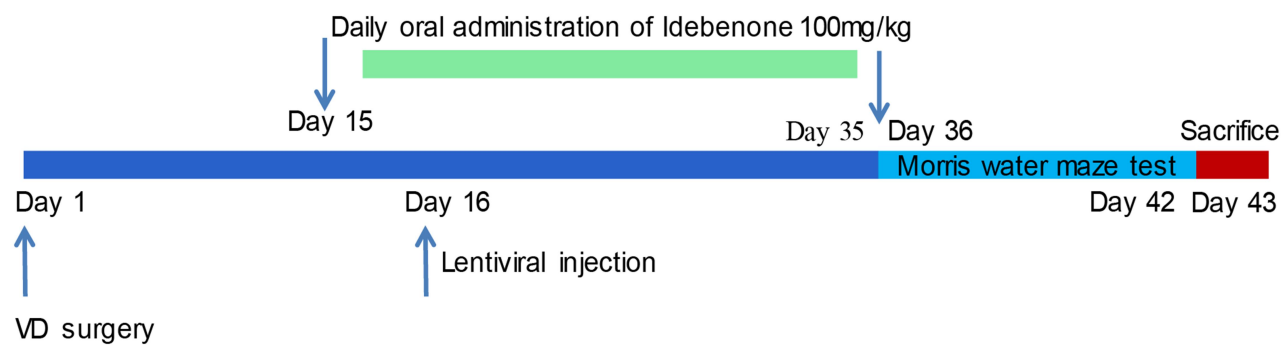

Figure I Flow chart of the animal experiment. 
hematoxylin staining solution (Sigma-Aldrich, Merck KGaA, Darmstadt, Germany) for $10 \mathrm{~min}$. The excessive hematoxylin dyeing solution was removed with distilled water. The tissue sections were washed with ultrapure water, stained with eosin (Sigma-Aldrich) for $3 \mathrm{~min}$, dehydrated with different concentrations of ethanol, soaked in xylene, dried, and sealed with neutral resin. Tissue sections were observed under an upright fluorescent microscope (BX53, Olympus, Tokyo, Japan).

\section{Nissl Staining}

Paraffin-embedded sections $(7 \mu \mathrm{m})$ were dewaxed and hydrated. After washing with distilled water, the sections were stained with $0.25 \%$ toluidine blue (Shanghai Zeye Biotechnology Co., Ltd., Shanghai, China) at $60^{\circ} \mathrm{C}$ for 3 $\mathrm{h}$. The remaining dyeing was washed away with ultrapure water, and then the sections were washed with 95\% ethanol, dehydrated with absolute ethanol, cleared with xylene, and sealed with neutral gum. Finally, the sections were observed, and images were captured under a fluorescence microscope. The images were processed by ImageJ software (USA) to count the number of CA1 neurons per $0.1 \mathrm{~m}^{2}$ and calculate the average number of positive cells.

\section{Microarray Analysis}

The effect of Idebenone treatment on expression of miR in the hippocampus of rats was analyzed using SurePrint Rat miR Microarrays (Agilent Technology Co., Ltd., Beijing, China). Three repeated experiments were carried out for each group. The procedures included pre-hybridization, hybridization, washing and imaging. Agilent DNA Microarray Scanner (P/N G2565BA) equipped with extended dynamic range software was utilized for scanning and image analysis according to the Agilent miR Microarray System with miR Complete Labeling and Hyb Kit Protocol manual (Version 2.0 P/N G417090,011). Feature Extraction Software 10.5 was used for data extraction from raw microarray image files using the miRNA_105_Dec08 FE protocol. GeneSpring GX (Version 10.0) software was adopted for data visualization and analysis.

\section{Western Blot (WB) Analysis}

Protein was extracted from cells or tissues on ice with RIPA lysis buffer (Beyotime Biotechnology Co., Ltd, Shanghai, China) for $20 \mathrm{~min}$. The supernatant was obtained by centrifuging at $4^{\circ} \mathrm{C}$ at $12,000 \times \mathrm{g}$ for 20 min. The bicinchoninic acid kit (Thermo Fisher
Scientific Inc., Waltham, MA, USA) was used to quantify the protein concentration. An equal amount of protein (50 $\mu \mathrm{g})$ was separated by electrophoresis and transferred to nitrocellulose membrane. After a 2-h blocking with 5\% skim milk, the membranes were incubated with primary antibodies against RSK2 (1:1000, ab32062, Abcam), Phospho IкB $\alpha$ (Ser32) (1:1000, \#2859, CST, Beverly, MA, USA), IкB $\alpha$ (1:1000, \#4812, CST), Phospho-NF

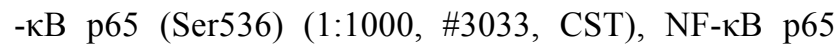
(1:1000, \#8242, CST), and GAPDH (1:1000, ab181602, Abcam) overnight at $4^{\circ} \mathrm{C}$. Then, the membrane was incubated with goat anti-rabbit IgG H\&L (HRP) (1:10,000, ab205718, Abcam) for $1 \mathrm{~h}$. Protein bands were visualized using an enhanced chemiluminescence kit (Beyotime). The protein bands were analyzed by ImageJ software, and the expression of target protein was calculated by comparing the gray value of each group with the GAPDH.

\section{Reverse Transcription Quantitative Polymerase Chain Reaction (RT-qPCR)}

TRIzol reagent (Invitrogen, Carlsbad, CA, USA) was used for extraction of total RNA containing miR and mRNA from cells and tissues. TaqMan ${ }^{\mathrm{TM}}$ Advanced miRNA cDNA Synthesis Kit or SuperScript ${ }^{\mathrm{TM}}$ IV First-Strand Synthesis System (Thermo Fisher) was adopted for cDNA synthesis. RT-qPCR was performed with $2 \times$ SYBR Green qPCR Mix (Invitrogen) on an ABI 7900HT qPCR system (Thermo Fisher). The expression was determined using the ${ }^{-}{ }^{\Delta \Delta \mathrm{CT}}$ method with GAPDH as the internal reference of $\mathrm{mRNA}$ and $5 \mathrm{~S}$ as the internal reference of miR. The primer sequences used in the experiment are shown in Table 1.

\section{Dual-Luciferase Reporter Gene Assay}

RSK2 3'UTR sequence containing a potential binding site to miR-216a (WT-RSK2) and mutated binding site (MTRSK2) were obtained from TargetScan. The above sequences were inserted into pmirGLO vector (Promega, MI, USA) to produce the report plasmid. We cotransfected the luciferase reporter plasmids with miR216a mimic or their controls (Shanghai GenePharma Co., Ltd., Shanghai, China) into 293 T cells (Fenghuishengwu, Changsha, Hunan, China) using Lipofectamine 2000 (Thermo Fisher). After 2 days of incubation, the dualluciferase assay system (Promega) was adopted to determine the luciferase activity. 
Table I Sequence Used for RT-qPCR

\begin{tabular}{|c|c|c|}
\hline Gene & Forward Primer (5'-3') & Reverse Primer $\left(5^{\prime}-3^{\prime}\right)$ \\
\hline$m i R-216 a$ & TAATCTCAGCTGGCAACTGTG & GAACATGTCTGCGTATCTC \\
\hline RSK2 & TAACCGCAGAGGTCACACTCAG & CTCAGAAACTGTGGCATCCCGA \\
\hline FZD4 & ACTTTCACGCCGCTCATCCAGT & TCTCAGGACTGGTTCACAGCGT \\
\hline NAAI5 & TGGTCAGCCATCCATTGCTCTG & CCATCCACCTGGCAGCTTCTTT \\
\hline RSBNI & TGTAGGTCTCAGAGCGATGACG & GTTCACTTGTCCGAGGTAGGTAC \\
\hline GAPDH & CATCACTGCCACCCAGAAGACTG & ATGCCAGTGAGCTTCCCGTTCAG \\
\hline $5 S$ & CTCGCTTCGGCAGCACAT & TTTGCGTGTCATCCTTGCG \\
\hline
\end{tabular}

Abbreviations: RT-qPCR, reverse transcription quantitative polymerase chain reaction; miR, microRNA; RSK2, ribosomal S6 kinase 2; FZD4, frizzled 4; NAAI5, N-alphaacetyltransferase I5; RSBNI, round spermatid basic protein I; GAPDH, glyceraldehyde-3-phosphate dehydrogenase.

\section{Statistical Analysis}

GraphPad prism 8.0 (GraphPad, San Diego, CA, USA) was used to analyze data. All the experiments were repeated three times independently, and the data were in accordance with normal distribution and showed as mean \pm standard error of mean (SEM). Unpaired $t$-set was adopted for comparison between two groups, and one-way or two-way analysis of variance (ANOVA) was adopted for comparison among multi-groups, followed by Tukey's post hoc test. The $p<$ 0.05 meant a statistically significant difference.

\section{Results}

\section{Idebenone Ameliorates Brain Injury in VD Rats}

The VD rat model was established by bilateral CCA occlusion and treated with Idebenone. The learning ability of rats was detected by analyzing the escape latency, and the memory ability of rats was detected by analyzing the times crossing the platform (Figure 2A and B). The escape latency of sham-operated rats decreased evidently on the 2nd day and the 3rd day, and remained at a low value from the 4th to the 5th day, which indicated that the rats in the sham group had better learning ability. The escape latency of VD rats increased from the 2nd day to the 5th day, indicating that the learning ability of VD rats was significantly decreased. Compared with VD group, the escape latency of Idebenone-treated rats was notably reduced from the 3 rd to the 5th day, which indicated that Idebenone treatment improved the learning ability of VD rats. In the platform crossing analysis, the time of rats crossing platform in VD group was reduced, which proved that VD modeling led to the damage of spatial memory ability of rats. However, after Idebenone treatment, the number of times the rats crossed the platform was clearly reduced. However, there was no notable difference in swimming speed among the groups, indicating that abnormal behaviors of VD rats were not related to dyskinesia (Figure 2C).

Idebenone can inhibit oxidative stress and inflammatory damage to protect the body, ${ }^{18-20}$ which are the causes of $\mathrm{VD},{ }^{21,22}$ so we speculated that the therapeutic effect of Idebenone was elicited on VD rats through suppressing oxidative stress and inflammatory damage. We detected the expression of MDA, SOD, IL- 6 and TNF- $\alpha$ in hippocampus by ELISA. VD modeling resulted in a significant increase in the levels of MDA, IL-6 and TNF- $\alpha$, and a significant decrease in the concentration of SOD. The levels of these indicators were reversed after Idebenone treatment (Figure 2D) (all $p<0.05$ ).

To intuitively observe the nerve injury of rats, we also carried out histochemical staining. HE staining (Figure 2E) was performed on the hippocampal cortex of rats. The neurons in VD rats showed obvious disorder of arrangement and structural loss. The space between neurons was enlarged, and the nucleus showed shrinkage and unclear structure. Idebenone treatment can significantly reduce the damage of neurons induced by VD. Nissl staining of hippocampal CA1 region showed that Nissl bodies in VD rats were markedly reduced, while significantly elevated after Idebenone treatment (Figure 2F).

\section{Inhibition of miR-2I6a Attenuates the Improvement of Idebenone on Learning and Spatial Memories in VD Rats}

To study the molecular mechanism of Idebenone on VD rats, we performed microarray analysis of hippocampal tissues in VD rats and those treated with Idebenone and showed the top 10 differentially expressed miRs (Figure 3A). After Idebenone treatment, miR-216a in the hippocampus of rats was increased. According to the previous report, ${ }^{14}$ miR-216a could reduce 


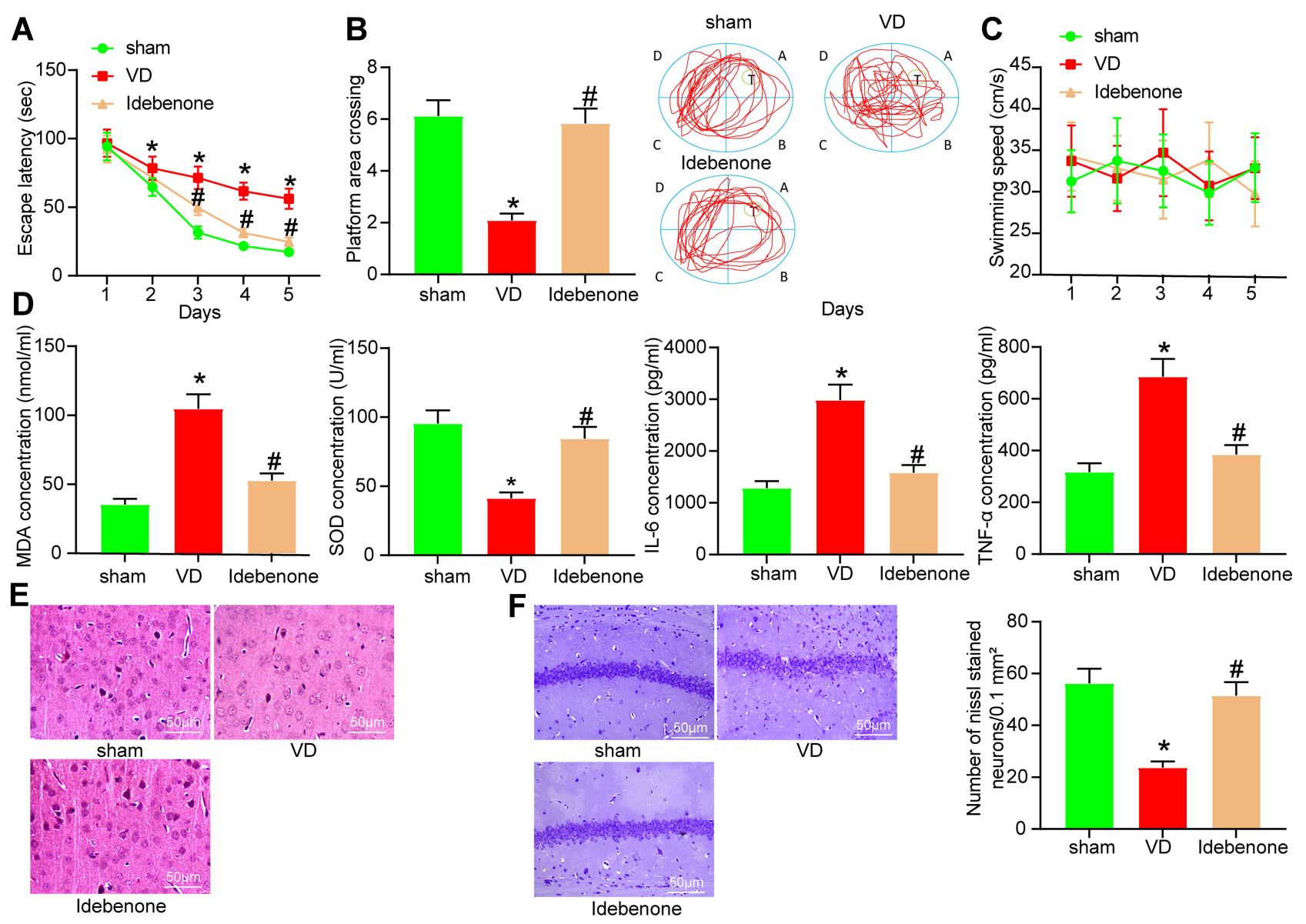

Figure 2 Idebenone ameliorates brain injury in VD rats. (A) The escape latency test examined by MWM test. (B) The spatial memory ability of rats tested by platform crossing experiment. (C) Swimming speed of rats. (D) The concentrations of oxidative stress- and inflammation-related indexes in hippocampus detected by ELISA. (E) The neuronal damage in the hippocampal cortex examined by HE staining. (F) The neuronal damage in hippocampal CAI area of rats measured by Nissl staining. $\mathrm{N}=10$. All experiments were repeated three times independently. The results were shown as mean \pm SEM or representative pictures. Data in panels $\mathbf{B} / \mathbf{D} / \mathbf{F}$ were analyzed by one-way ANOVA, and data in panels A/C were analyzed by two-way ANOVA. ${ }^{*} p<0.05$, vs the sham; \#p $<0.05$, vs the VD.

neuroinflammation. miR-216a also plays an antiinflammatory role in lung injury and diabetic retinopathy, ${ }^{23,24}$ and inhibits oxidative stress in asthma. ${ }^{25}$ Therefore, we speculated that the therapeutic effect of Idebenone on VD depended on miR-216a. While the expression of miR-216a in hippocampal tissues of rats in the shamoperated and VD groups was detected by RT-qPCR assay, we found that its expression was significantly reduced in VD rats (Figure 3B).

miR-216a inhibitor encapsulated by lentivirus was injected into the brain ventricle of rats treated with Idebenone. The corresponding Lv-NC vector was used as control. The escape latency of Lv inhibitor group was increased from the 3 rd to the 5 th day (Figure 3C), while the times of crossing the platform were significantly lowered (Figure 3D). There was no notable difference in swimming speed between the two groups (Figure 3E).
Inhibition of miR-2/6a Weakens the Inhibitory Effect of Idebenone on

\section{Neuroinflammation and Oxidative Stress in VD Rats}

In the Lv-inhibitor group, miR-216a expression in hippocampus was decreased (Figure 4A). ELISA assay results revealed that the concentration of MDA, IL- 6 , and TNF- $\alpha$ was clearly increased, while SOD was decreased (Figure 4B). HE staining showed that the protective effect of Idebenone on hippocampal cortical neurons was evidently inhibited (Figure 4C). Nissl staining revealed that the Nissl bodies were significantly lowered after inhibition of miR-216a (Figure 4D).

\section{miR-2I6a Targets RSK2}

The target genes of miR-216a were selected from TargetScan, miRDB and mirWalk (Figure 5A). FZD4, 

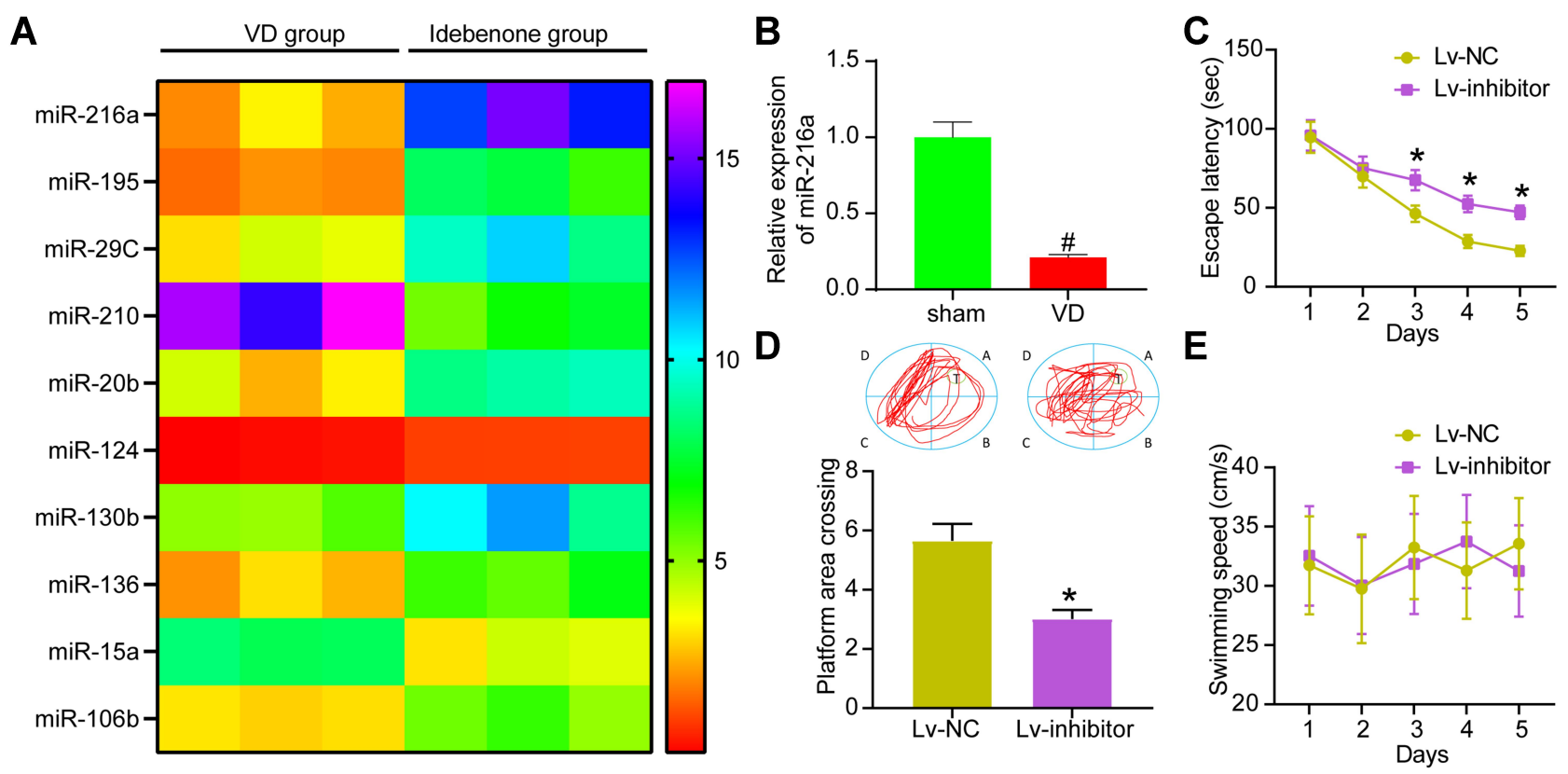

Figure 3 Inhibition of miR-2I6a attenuates the improvement of Idebenone on learning and spatial memory abilities in VD rats. (A) The differentially expressed miRs in hippocampus of VD group and Idebenone group screened by microarray analysis. (B) Detection of miR-2I6a expression in VD rats by RT-qPCR. (C) The learning ability of rats was detected by escape latency test. (D) The spatial memory ability of rats was detected by platform crossing experiment. (E) The changes of swimming speed. $\mathrm{N}=10$. All the experiments were repeated three times independently. The results showed that the average value was \pm SEM. Unpaired $t$-test was used for comparison in panel B/D, and two-way ANOVA was used for comparison in panels C/E. ${ }^{*} p<0.05$, vs Lv-NC; \#p $<0.05$, vs sham-operated rats.
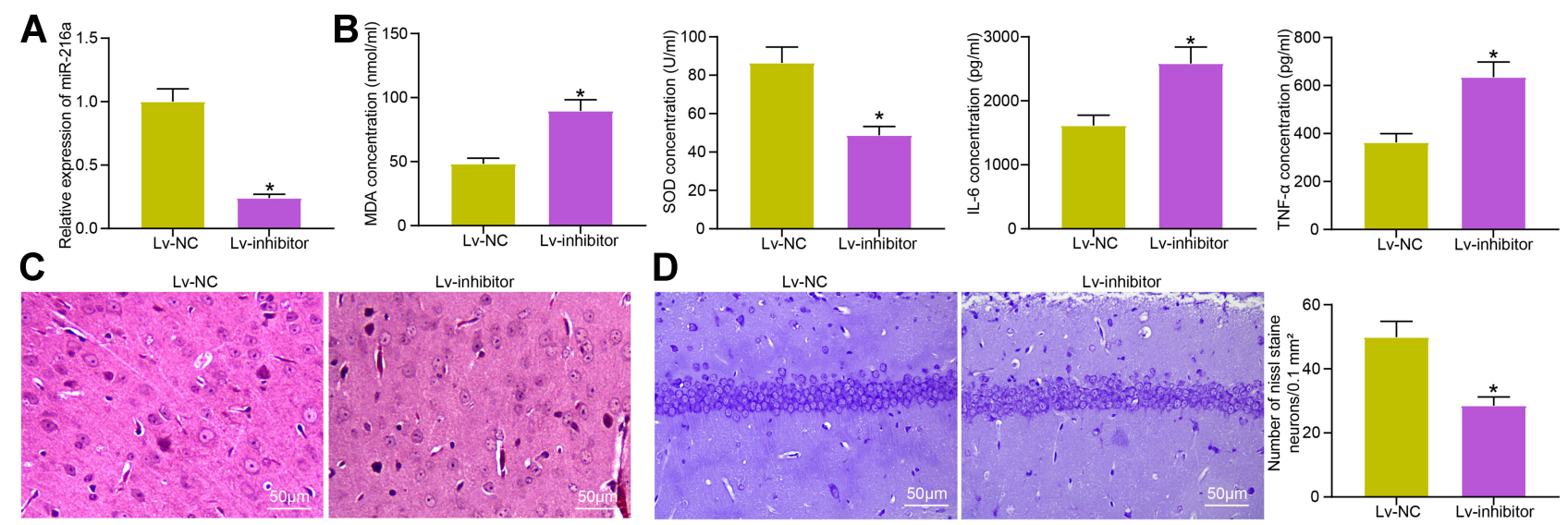

Figure 4 Inhibition of miR-2I6a weakens the inhibitory effect of Idebenone on neuroinflammation and oxidative stress in VD rats. (A) The miR-2I6a expression in hippocampus of rats after injection determined by RT-qPCR. (B) The concentration of inflammation- and oxidative stress-related indicators in the hippocampus examined by ELISA. (C) The neuronal damage in the hippocampal cortex evaluated by HE staining. (D) The damage of neurons observed by Nissl staining. $\mathrm{N}=10$. All the experiments were repeated three times independently. The results showed that the average value was \pm SEM. Unpaired $t$-test was used for comparison between two groups. $* p<0.05$, vs Lv-NC.

NAA15, RSBN1 and RPS6KA3 (also known as RSK2) were in the intersection of the three databases (Figure 5B). RT-qPCR elicited that only RSK2 expression had significant differences in the hippocampus of rats (Figure 5C).

We detected the expression of RSK2 in hippocampal tissues of rats in sham-operated and VD groups by RT-
qPCR assay. Its expression was significantly elevated in hippocampal tissues of VD rats (Figure 5D). The potential binding sites of miR-216a and RSK2 were obtained in TargetScan, and the RSK2-MUT sequence was constructed by mutating the binding sites (Figure $5 \mathrm{E}$ ). miR-216a mimic clearly inhibited the luciferase activity of RSK2-WT, but had no significant effect on that of RSK2-MUT (Figure 5F). 

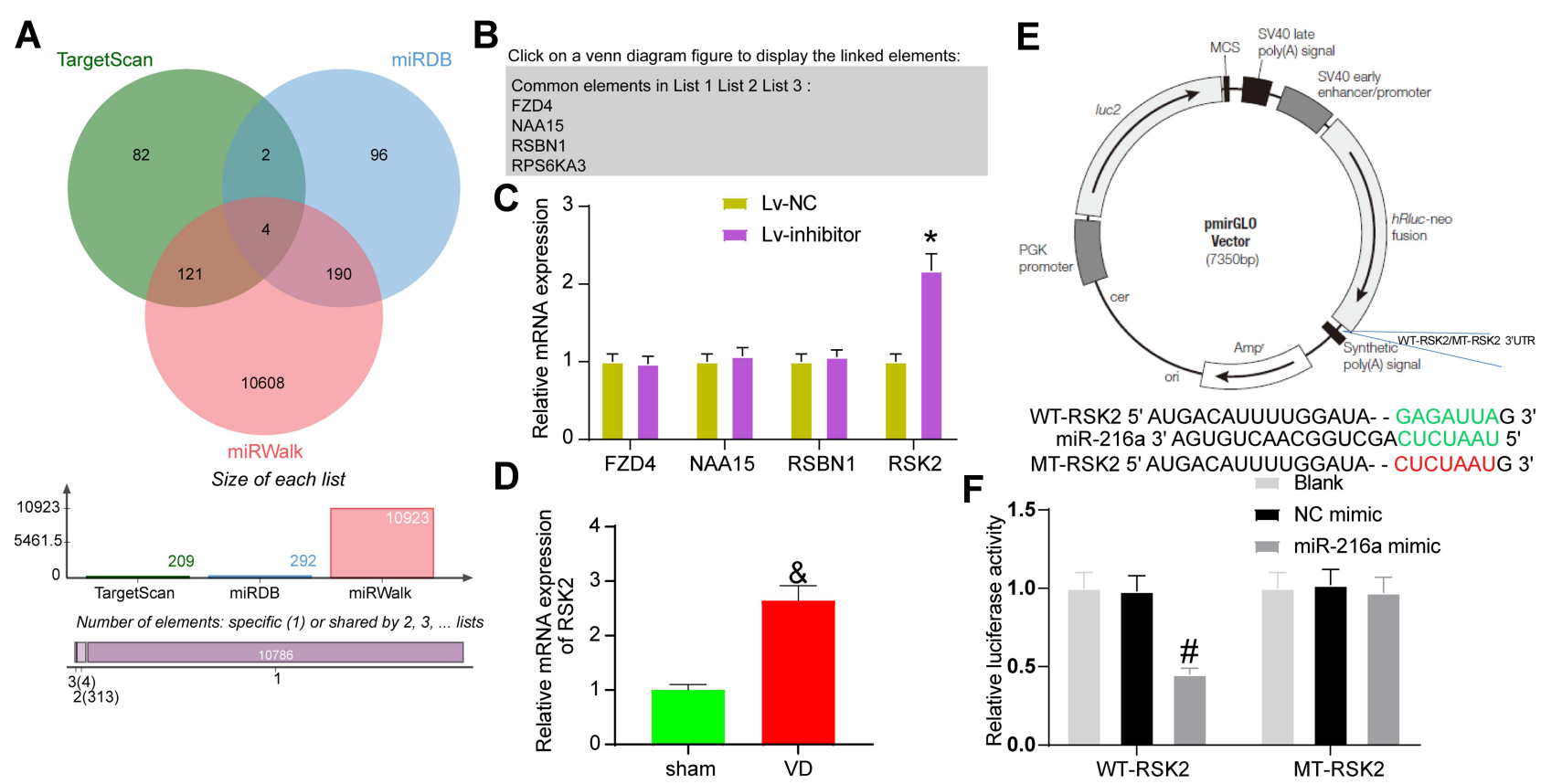

WT-RSK2 5' AUGACAUUUUGGAUA- - GAGAUUAG 3 miR-216a 3' AGUGUCAACGGUCGACUCUAAU 5'
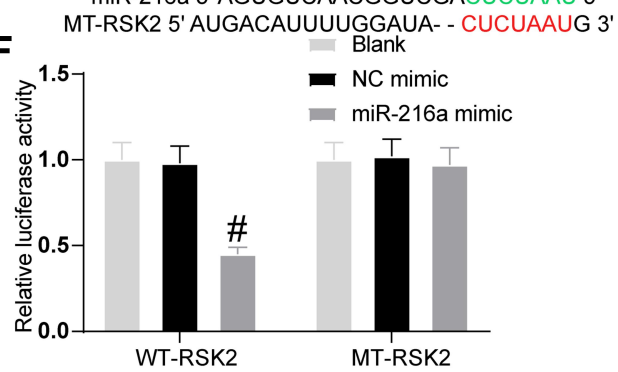

Figure 5 miR-216a targets RSK2. (A) The potential target genes of miR-2I6a predicted in several bioinformatics websites. (B) The intersection of potential target genes. (C) The expression of potential target genes in rat hippocampus examined by RT-qPCR. (D) Detection of RSK2 expression in VD rats by RT-qPCR. (E) pmirGLO plasmid mapping and sequences for RSK2-WT and RSK2-MUT. (F) The luciferase activity of WT-RSK2 and MT-RSK2 tested by dual-luciferase assay. All the experiments were repeated three times independently, and the results were averaged. Unpaired $t$-test was used for comparison between two groups in panel D. Two-way ANOVA was used for comparison in panels C/F. \&p $<0.05$, vs sham-operated rats; ${ }^{*} p<0.05$, vs the Lv-NC; \#p<0.05, vs the NC mimic; \&p<0.05, vs sham-operated rats.

\section{Overexpression of RSK2 Attenuates the Therapeutic Effect of Idebenone on VD Rats via the $I \kappa B \alpha / N F-\kappa B$ Axis}

The rats treated with Idebenone were injected with lentivirus LV-RSK2 into the ventricles, and the rats injected with LvNC served as controls. The learning and spatial memory abilities of rats injected with Lv-RSK2 were notably decreased (Figure 6A and B). The specific manifestations were the prolongation of escape latency and the decrease of crossing platform times. There was no significant effect of lentivirus injection on the motor ability of rats (Figure 6C).

According to the previous report, ${ }^{26} \mathrm{RSK} 2$ can promote the phosphorylation of $\mathrm{I} \kappa \mathrm{B} \alpha$ and $\mathrm{NF}-\kappa \mathrm{B}$. WB showed that RSK2 and P-IкB $\alpha, \mathrm{I} \kappa \mathrm{B} \alpha, \mathrm{p} 65$, p-p65 levels were elevated in the hippocampus of rats (Figure 6D). The expression of RSK2 and phosphorylation of $\mathrm{I} \kappa \mathrm{B} \alpha$ and NF- $\mathrm{NB}$ were significantly enhanced in rats injected with Lv-RSK2. Consequently, the levels of MDA, IL-6 and TNF- $\alpha$ in hippocampus were clearly increased, while SOD was decreased (Figure 6E).

HE staining implied that neurons in rats injected with Lv-RSK2 showed obvious pathological phenomena, such as uneven arrangement, incomplete structure and increased gap (Figure 6F). Nissl staining elicited that RSK2 overexpression reduced Nissl bodies in CA1 area of hippocampus (Figure 6G).

\section{Discussion}

VD is featured by a progressive deterioration of memory and cognitive functions caused by cerebrovascular diseases. ${ }^{1}$ In recent decades, many studies have been proposed to the therapeutic potential of Idebenone in the treatment of neurodegenerative diseases. ${ }^{8,27}$ This study revealed that Idebenone exerted therapeutic effects on VD rats by repressing oxidative stress and neuroinflammation.

Emerging evidence has highlighted the critical roles of oxidative stress and neuroinflammation in the cognitive impairment in VD patients. $^{22}$ Idebenone can attenuate oxidative stress and inflammatory reaction induced by titanium dioxide nanoparticles in the rat liver. ${ }^{19}$ Idebenone shows significant neuroprotective and antioxidant activities in pilocarpine-induced seizures. ${ }^{18}$ Accordingly, we speculated that Idebenone might exert therapeutic effects on VD rats by reducing oxidative stress and inflammatory reaction. Therefore, we detected the indicators relating oxidative stress and inflammation in the hippocampus of rats. After Idebenone treatment, the levels of MDA, IL-6, TNF- $\alpha$ were decreased significantly, and the concentration of SOD was increased. Consistently, 

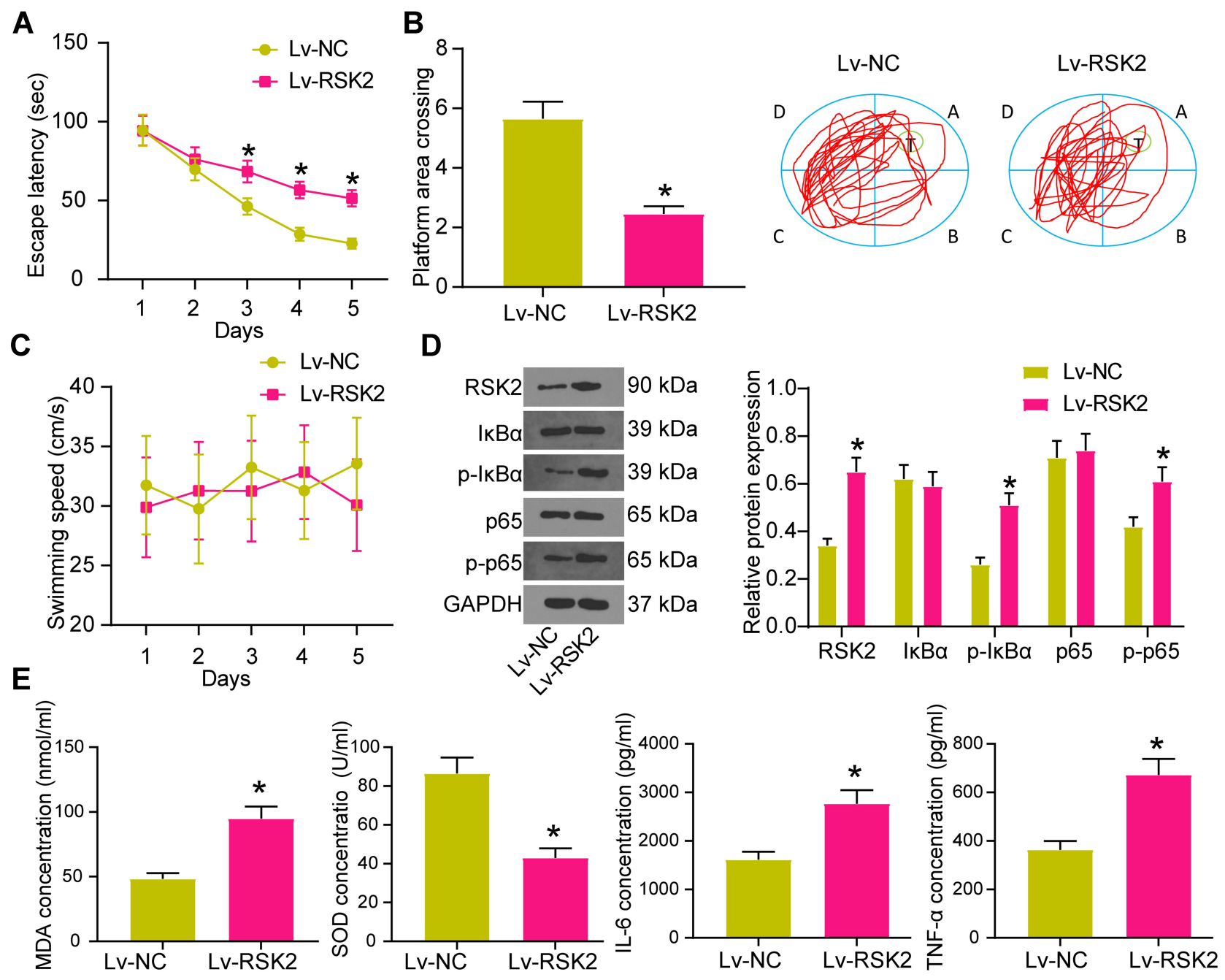

$\mathbf{F}$

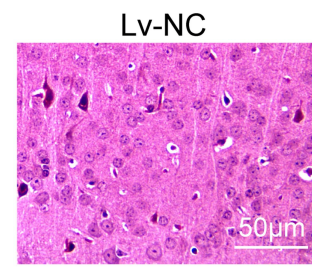

LV-RSK2

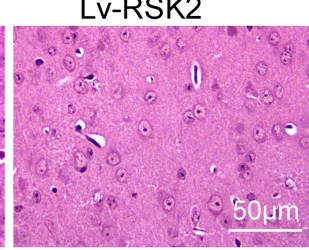

G

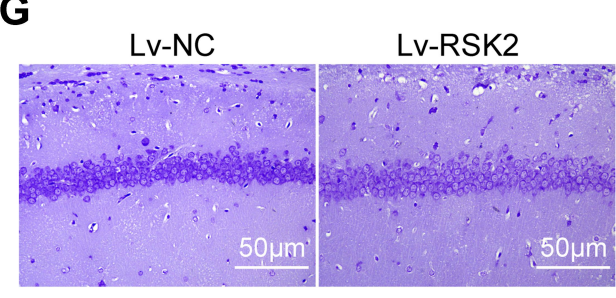

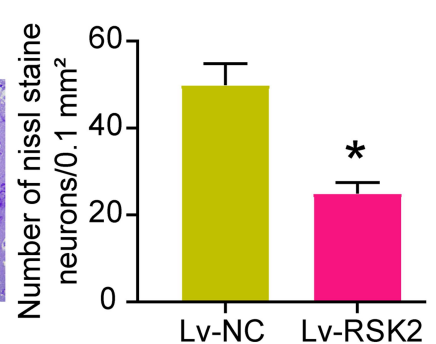

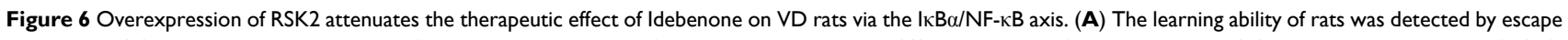
latency test. (B) The spatial memory ability of rats was detected by platform crossing experiment. (C) The changes of swimming speed. (D) the protein expression of RSK2, I $\mathrm{B} \alpha, \mathrm{P}-\mathrm{I} \mathrm{\kappa} \mathrm{B} \alpha$, p65 and p-p65 in the hippocampus of rats detected by WB. (E) The levels of oxidative stress- and inflammation-related factors in hippocampus tissue measured by ELISA. (F) the neuronal damage in the hippocampal cortex evaluated by HE staining. (G) the neuronal damage in CAI area of hippocampus examined by Nissl staining. $\mathrm{N}=10$. All experiments were repeated three times independently. The results were shown as mean \pm SEM or representative pictures. Unpaired $t$-test was used for comparison in panels $\mathbf{B} / \mathbf{E} / \mathbf{G}$, and two-way ANOVA was used for comparison in panels A/C/D. $* p<0.05$, vs the Lv-NC.

Marcus Kernt et al have revealed that Idebenone inhibits oxidative stress and apoptosis of astrocytes in human optic nerve head by stabilizing Bax/Bcl-2 ratio. $^{28}$ Yan et al have clarified that Idebenone can attenuate neuroinflammation and regulate microglial polarization in a mouse model of Parkinson's disease. ${ }^{29}$ Moreover, Idebenone treatment can notably reduce the damage of neurons and increase Nissl bodies. Taken together, Idebenone mitigated brain injury in the rat model of VD.

Then, we investigated the molecular mechanism of Idebenone in VD. We analyzed the hippocampal tissues of rats in the VD group and Idebenone group by microarray 
analysis and screened the top ten miRs with differential expression. We found that the miR-216a expression in hippocampus was significantly elevated after Idebenone treatment. miR-216a is implicated in the tumorigenesis and progression of diverse human malignancies, such as renal cell carcinoma, breast cancer and pancreatic cancer. ${ }^{30-32}$ However, the role of miR-216a in VD remains unknown yet. Intriguingly, the existing evidence indicates that elevated miR-216a can exert neuroprotective effects on cerebral ischemia injury. ${ }^{14}$ miR-216a can attenuate lipopolysaccharide-induced inflammatory injury via the JAK2/STAT3 and $\mathrm{NF}-\kappa \mathrm{B}$ signaling. ${ }^{23}$ miR-216a-5p protects $16 \mathrm{HBE}$ cells from $\mathrm{H}_{2} \mathrm{O}_{2}$-induced oxidative stress by targeting the HMGB1/NF$\kappa \mathrm{B}$ pathway. ${ }^{25} \mathrm{We}$ thus speculated that the therapeutic effects of Idebenone on VD rats depend on increasing miR-216a expression. To verify our hypothesis, we injected miR-129 inhibitor into the ventricles of rats treated with Idebenone. The results exhibited that miR-216a inhibitor not only attenuated the improving effect of Idebenone on learning and spatial memory abilities, but also weakened the inhibitory effect of Idebenone on neuroinflammation and oxidative stress on VD rats. In brief, inhibition of miR-216a weakened the therapeutic effects of Idebenone on VD rats.

Thereafter, we screened the target genes of miR-216a through the database and detected their mRNA expression in the hippocampus of VD rats delivered with LV-NC or Lv-inhibitor. We found that only RSK2 showed significant differential expression. The binding relationship between miR-216a and RSK2 was verified using dual-luciferase reporter gene assay. Activation of RSK2 promotes the inflammatory process of fibroblast-like synoviocytes. ${ }^{33}$ RSK2 is activated when microglia and THP1 monocytes are exposed to $\beta$-amyloid protein, which is the main component of Alzheimer's plaques. ${ }^{34}$ We conducted functional rescue experiments to prove that Idebenone improved the symptoms of VD rats via targeting miR-216a/RSK2 axis. The results exhibited that overexpression of RSK2 attenuated the therapeutic effects of Idebenone on VD. RSK2 can promote the phosphorylation of $\mathrm{I} \kappa \mathrm{B} \alpha$, thus facilitating the activation and transferring of p65 and p50 of NF- $\kappa \mathrm{B}$ to the nucleus. ${ }^{26} \mathrm{NF}-\kappa \mathrm{B}$ is a redox-sensitive transcription factor family, which has binding sites in the promoter region of amyloidogenesis and inflammation-related genes. $^{35}$ The activation of NF- $\mathrm{KB}$ results in the elevation of pro-inflammatory cytokines and proteins, which consequently causes inflammatory reaction and cell death. ${ }^{36}$ Since NF- $\kappa$ B is implicated in the physiological inflammatory processes, it is extensively accepted as a potential target of the inflammation-based therapy for Alzheimer's disease. $^{37}$ Yao et al have also demonstrated that NF- $\kappa$ B pathway may be involved in the mechanism of triptolide improving cognitive dysfunction in VD rats. ${ }^{38}$ The miR$216 \mathrm{a} / \mathrm{RSK} 2$ axis regulated the therapeutic effects of Idebenone by mediating the $\mathrm{I} \kappa \mathrm{B} \alpha / \mathrm{NF}-\kappa \mathrm{B}$ pathway.

\section{Conclusion}

To sum up, Idebenone ameliorated oxidative stress and neuroinflammation in VD rats via the miR-216a/RSK2/I $\kappa \mathrm{B} \alpha /$ $N F-\kappa B$ axis. Our current data indicate that Idebenone has therapeutic effects on neurodegenerative diseases as a novel anti-inflammatory and antioxidant stress agent. In the future, we shall carry out more prospective studies to verify the safety and feasibility of Idebenone in the treatment of VD.

\section{Funding}

There is no funding to report.

\section{Disclosure}

The authors declare no potential conflicts of interest.

\section{References}

1. Iadecola C. The pathobiology of vascular dementia. Neuron. 2013;80 (4):844-866.

2. Kales HC, Gitlin LN, Lyketsos CG. Assessment and management of behavioral and psychological symptoms of dementia. BMJ. 2015;350 (mar02 7):h369. doi:10.1136/bmj.h369

3. Nguyen DH, Cunningham JT, Sumien N. Estrogen receptor involvement in vascular cognitive impairment and vascular dementia pathogenesis and treatment. Geroscience. 2020. doi:10.1007/s11357-020-00263-4

4. Mulugeta E, Molina-Holgado F, Elliott MS, et al. Inflammatory mediators in the frontal lobe of patients with mixed and vascular dementia. Dement Geriatr Cogn Disord. 2008;25(3):278-286. doi:10.1159/000118633

5. Luca M, Luca A, Calandra C. The role of oxidative damage in the pathogenesis and progression of alzheimer's disease and vascular dementia. Oxid Med Cell Longev. 2015;2015:504678. doi:10.1155/ 2015/504678

6. Belkhelfa M, Beder N, Mouhoub D, et al. The involvement of neuroinflammation and necroptosis in the hippocampus during vascular dementia. J Neuroimmunol. 2018;320:48-57. doi:10.1016/j. jneuroim.2018.04.004

7. Farooq MU, Min J, Goshgarian C, Gorelick PB. Pharmacotherapy for vascular cognitive impairment. CNS Drugs. 2017;31(9):759-776. doi:10.1007/s40263-017-0459-3

8. Montenegro L, Turnaturi R, Parenti C, Pasquinucci L. Idebenone: novel strategies to improve its systemic and local efficacy. Nanomaterials. 2018;8(2). doi:10.3390/nano8020087

9. Meier T, Buyse G. Idebenone: an emerging therapy for friedreich ataxia. J Neurol. 2009;256(Suppl 1):25-30. doi:10.1007/s00415-0091005-0

10. Wang H, Li L, Jia K, et al. Idebenone protects mitochondrial function against amyloid beta toxicity in primary cultured cortical neurons. Neuroreport. 2020;31(15):1104-1110. doi:10.1097/WNR.0000 000000001526 
11. Zhang ZW, Xu XC, Liu T, Yuan S. Mitochondrion-permeable antioxidants to treat ROS-burst-mediated acute diseases. Oxid Med Cell Longev. 2016;2016:6859523. doi:10.1155/2016/6859523

12. Nappi G, Bono G, Merlo P, et al. Long-term idebenone treatment of vascular and degenerative brain disorders of the elderly. Arch Gerontol Geriatr. 1992;15(3):261-269. doi:10.1016/0167-4943(92) 90061-8

13. Yang TT, Liu CG, Gao SC, Zhang Y, Wang PC. The serum exosome derived MicroRNA-135a, $-193 \mathrm{~b}$, and -384 were potential alzheimer's disease biomarkers. Biomed Environ Sci. 2018;31(2):87-96. doi:10.3967/bes2018.011

14. Tian YS, Zhong D, Liu QQ, et al. Upregulation of miR-216a exerts neuroprotective effects against ischemic injury through negatively regulating JAK2/STAT3-involved apoptosis and inflammatory pathways. J Neurosurg. 2018;130(3):977-988. doi:10.3171/2017.5. JNS163165

15. Yang X, Zhang M, Wei M, Wang A, Deng Y, Cao H. MicroRNA216a inhibits neuronal apoptosis in a cellular parkinson's disease model by targeting Bax. Metab Brain Dis. 2020;35(4):627-635. doi:10.1007/s11011-020-00546-X

16. Jian WX, Zhang Z, Zhan JH, et al. Donepezil attenuates vascular dementia in rats through increasing BDNF induced by reducing HDAC6 nuclear translocation. Acta Pharmacol Sin. 2020;41 (5):588-598. doi:10.1038/s41401-019-0334-5

17. Ai J, Sun LH, Che H, et al. MicroRNA-195 protects against dementia induced by chronic brain hypoperfusion via its anti-amyloidogenic effect in rats. $J$ Neurosci. 2013;33(9):3989-4001. doi:10.1523/ JNEUROSCI.1997-12.2013

18. Ahmed MA. Neuroprotective effects of idebenone against pilocarpine-induced seizures: modulation of antioxidant status, DNA damage and $\mathrm{Na}(+), \quad \mathrm{K}(+)$-ATPase activity in rat hippocampus. Neurochem Res. 2014;39(2):394-402. doi:10.1007/ s11064-014-1236-z

19. Fadda LM, Hagar H, Mohamed AM, Ali HM. Quercetin and idebenone ameliorate oxidative stress, inflammation, DNA damage, and apoptosis induced by titanium dioxide nanoparticles in rat liver. Dose Response. 2018;16(4):1559325818812188. doi:10.1177/ 1559325818812188

20. Peng J, Wang H, Gong Z, et al. Idebenone attenuates cerebral inflammatory injury in ischemia and reperfusion via dampening NLRP3 inflammasome activity. Mol Immunol. 2020;123:74-87. doi:10.1016/ j.molimm.2020.04.013

21. Bhatia P, Singh N. Tadalafil ameliorates memory deficits, oxidative stress, endothelial dysfunction and neuropathological changes in rat model of hyperhomocysteinemia induced vascular dementia. Int J Neurosci. 2020;1-13. doi:10.1080/00207454.2020.1817009

22. Wang L, Yang JW, Lin LT, et al. Acupuncture attenuates inflammation in microglia of vascular dementia rats by inhibiting miR-93mediated TLR4/MyD88/NF-kappaB signaling pathway. Oxid Med Cell Longev. 2020;2020:8253904.

23. Kong F, Sun Y, Song W, Zhou Y, Zhu S. MiR-216a alleviates LPS-induced acute lung injury via regulating JAK2/STAT3 and NF-kappaB signaling. Hum Cell. 2020;33(1):67-78. doi:10.1007/ s13577-019-00289-7

Neuropsychiatric Disease and Treatment

\section{Publish your work in this journal}

Neuropsychiatric Disease and Treatment is an international, peerreviewed journal of clinical therapeutics and pharmacology focusing on concise rapid reporting of clinical or pre-clinical studies on a range of neuropsychiatric and neurological disorders. This journal is indexed on PubMed Central, the 'PsycINFO' database and CAS, and
24. Liu Y, Xiao J, Zhao Y, et al. microRNA-216a protects against human retinal microvascular endothelial cell injury in diabetic retinopathy by suppressing the NOS2/JAK/STAT axis. Exp Mol Pathol. 2020;115:104445. doi:10.1016/j.yexmp.2020.104445

25. Chaoyang Y, Qingfeng B, Jinxing F. MiR-216a-5p protects $16 \mathrm{HBE}$ cells from $\mathrm{H} 2 \mathrm{O} 2$-induced oxidative stress through targeting HMGB1/ NF-kB pathway. Biochem Biophys Res Commun. 2019;508 (2):416-420. doi:10.1016/j.bbrc.2018.11.060

26. Peng C, Cho YY, Zhu F, et al. RSK2 mediates NF-\{kappa $\}$ B activity through the phosphorylation of IkappaBalpha in the TNF-R1 pathway. FASEB J. 2010;24(9):3490-3499. doi:10.1096/fj.09-151290

27. Jaber S, Polster BM. Idebenone and neuroprotection: antioxidant, pro-oxidant, or electron carrier? J Bioenerg Biomembr. 2015;47(1-2):111-118. doi:10.1007/s10863-014-9571-y

28. Kernt M, Arend N, Buerger A, et al. Idebenone prevents human optic nerve head astrocytes from oxidative stress, apoptosis, and senescence by stabilizing BAX/Bcl-2 ratio. J Glaucoma. 2013;22 (5):404-412. doi:10.1097/IJG.0b013e31824caf90

29. Yan A, Liu Z, Song L, et al. Idebenone alleviates neuroinflammation and modulates microglial polarization in LPS-stimulated BV2 cells and MPTP-induced parkinson's disease mice. Front Cell Neurosci. 2018;12:529. doi:10.3389/fncel.2018.00529

30. Cui Y, Wang J, Liu S, et al. miR-216a promotes breast cancer cell apoptosis by targeting PKCalpha. Fundam Clin Pharmacol. 2019;33 (4):397-404. doi:10.1111/fcp.12481

31. Wang W, Zhao E, Yu Y, Geng B, Zhang W, Li X. MiR-216a exerts tumor-suppressing functions in renal cell carcinoma by targeting TLR4. Am J Cancer Res. 2018;8(3):476-488.

32. Zhang Y, Tang X, Shi M, Wen C, Shen B. MiR-216a decreases MALAT1 expression, induces G2/M arrest and apoptosis in pancreatic cancer cells. Biochem Biophys Res Commun. 2017;483 (2):816-822. doi:10.1016/j.bbrc.2016.12.167

33. Sun C, Sun Y, Jiang D, et al. PDK1 promotes the inflammatory progress of fibroblast-like synoviocytes by phosphorylating RSK2 Cell Immunol. 2017;315:27-33. doi:10.1016/j.cellimm.2016.10.007

34. McDonald DR, Bamberger ME, Combs CK, Landreth GE. betaamyloid fibrils activate parallel mitogen-activated protein kinase pathways in microglia and THP1 monocytes. $J$ Neurosci. 1998;18 (12):4451-4460. doi:10.1523/JNEUROSCI.18-12-04451.1998

35. Ju Hwang C, Choi DY, Park MH, Hong JT. NF-kappaB as a key mediator of brain inflammation in alzheimer's disease. CNS Neurol Disord Drug Targets. 2019;18(1):3-10. doi:10.2174/ 1871527316666170807130011

36. Li J, Wang H. miR-15b reduces amyloid-beta accumulation in SH-SY5Y cell line through targetting NF-kappaB signaling and BACE1. Biosci Rep. 2018;38:6.

37. Seo EJ, Fischer N, Efferth T. Phytochemicals as inhibitors of NFkappaB for treatment of alzheimer's disease. Pharmacol Res. 2018;129:262-273.

38. Yao P, Chen Y, Xu GH. Effect of triptolide on cognitive dysfunction in vascular dementia rats through SIRT1/NF-kappaB signaling pathway. Zhongguo Zhong Yao Za Zhi. 2019;44(16):3423-3428. doi:10.19540/j.cnki.cjemm.20180820.003

is the official journal of The International Neuropsychiatric Association (INA). The manuscript management system is completely online and includes a very quick and fair peer-review system, which is all easy to use. Visit http://www.dovepress.com/testimo-
nials.php to read real quotes from published authors.

Dovepress 